\title{
SCIENTIFIC REPORTS

\section{OPEN Estimation of Bladder Pressure and Volume from the Neural Activity of Lumbosacral Dorsal Horn Using a Long-Short-Term-Memory-based Deep Neural Network}

\begin{abstract}
Milad Jabbari \& Abbas Erfanian (i) *
In this paper, we propose a deep recurrent neural network (DRNN) for the estimation of bladder pressure and volume from neural activity recorded directly from spinal cord gray matter neurons. The model was based on the Long Short-Term Memory (LSTM) architecture, which has emerged as a general and effective model for capturing long-term temporal dependencies with good generalization performance. In this way, training the network with the data recorded from one rat could lead to estimating the bladder status of different rats. We combined modeling of spiking and local field potential (LFP) activity into a unified framework to estimate the pressure and volume of the bladder. Moreover, we investigated the effect of two-electrode recording on decoding performance. The results show that the two-electrode recordings significantly improve the decoding performance compared to single-electrode recordings. The proposed framework could estimate bladder pressure and volume with an average normalized root-mean-squared (NRMS) error of $14.9 \pm 4.8 \%$ and $19.7 \pm 4.7 \%$ and a correlation coefficient (CC) of $83.2 \pm 3.2 \%$ and $74.2 \pm 6.2 \%$, respectively. This work represents a promising approach to the real-time estimation of bladder pressure/volume in the closed-loop control of bladder function using functional electrical stimulation.
\end{abstract}

The lower urinary tract is responsible for the accumulation of urine (continence) and elimination of urine (micturition) at an appropriate time. Neurological disease or spinal cord injury (SCI) can disrupt the normal functions of the lower urinary tract, resulting in detrusor sphincter dyssynergia (DSD) and neurogenic detrusor hyperreflexia $(\mathrm{NDO})^{1}$. NDO is characterized by involuntary detrusor contractions during the filling phase even at small bladder volumes, while DSD consists of simultaneous contractions of the bladder and the urethral sphincter. DSD could lead to incomplete elimination of urine and high transient bladder pressures and NDO to low bladder capacity and incontinence.

Electrical stimulation has been used as an alternative approach to traditional methods for the treatment of neurogenic lower urinary tract dysfunction ${ }^{2}$. Many studies have demonstrated that reflex bladder inhibition can be achieved by electrical stimulation of the inhibitory pathways of pudendal nerve (PN) afferents to treat $\mathrm{NDO}^{3-5}$. Moreover, it has been shown that dorsal genital nerve stimulation can suppress undesired detrusor bladder contractions in patients with $\mathrm{NDO}^{6}$. It has also been demonstrated that stimulating and blocking of the pudendal nerves $^{7,8}$, high frequency electrical stimulation of the pudendal nerve and sacral root stimulation ${ }^{9}$, and stimulating and high frequency blocking of the sacral nerve roots ${ }^{10}$ can eliminate external urethral sphincter activation and restore bladder voiding. To produce micturition with reduced bladder-sphincter dyssynergia, a combination of low frequency and high frequency stimulation of the pelvic nerve has been investigated ${ }^{11}$. The low frequency stimulation of the distal pelvic nerve was provided to evoke bladder contraction, and proximal high frequency stimulation of the pelvic nerve was used to block afferent activation.

Electrical stimulation to restore bladder functions can be applied continuously or conditionally ${ }^{12-16}$. Continuous stimulation may increase the risk of tissue damage due to the energy deployed during the stimulation 
and the risk of implanted electrode corrosion and may lead to habituation of the spinal reflexes. Moreover, continuous stimulation requires high power consumption with respect to conditional stimulation.

To overcome the limitations of continuous stimulation, conditional stimulation can be employed, where the stimulation is applied when an impending bladder contraction is set to occur or the bladder requires voiding. To inhibit impeding bladder contraction by conditional stimulation, it is necessary to detect the onset of nascent hyperreflexive contractions.

Several methods for detecting bladder contractions and triggering stimulation have been reported. One common approach is to monitor intravesical pressure using artificial sensors ${ }^{17-23}$. However, there are a number of challenges facing artificial sensors that need to be addressed before clinical trials can be considered. These challenges are primarily related to invasiveness, artifacts from patient movement, abdominal pressure changes $^{24}$, material biocompatibility ${ }^{25}$, and related instruments' decreasing reliability over time ${ }^{26,27}$. Several alternative approaches have also been proposed to measure either the intravesical pressure or volume, using electromyography (EMG) of the external urethral sphincter ${ }^{6,28-30}$, electroneurography (ENG) of the pudendal nerve trunk using cuff-electrodes $^{5,31}$, pudendal nerve activity using penetrating intrafascicular electrodes ${ }^{32}$, and ENG of the pelvic nerve33 and sacral nerve roots ${ }^{33,34}$. However, several issues, such as a high degree of invasiveness, motion artifacts caused by organ movement, and low signal-to-noise ratios of electroneurograms, limit the chronic monitoring of intravesical pressure/volume. Moreover, ENG recordings from either the pudendal nerve $\mathrm{e}^{5,31}$, sacral root nerve $\mathrm{s}^{33,34}$ or pelvic nerves ${ }^{33}$ using cuff electrodes provide a whole-nerve action potential composite of several units in the nerve that also carries information from other sources.

Multi-channel recordings from dorsal root ganglia (DRG) ${ }^{27,35}$ or dissected intradural dorsal rootlets ${ }^{36}$ have also been considered as a potential alternative to pressure/volume estimation. The DRG is a cluster of sensory neurons that conveys information from the skin, muscles, and joints of the limbs and trunk to the spinal cord. Several studies have also demonstrated the feasibility of deriving limb-state estimates from the firing rates of primary afferent neurons recorded in $\mathrm{DRG}^{37-45}$. An important challenge facing DRG recordings is the long-term chronic recordings from DRGs. Recently, it has been demonstrated that the longest continuously tracked bladder afferent could last for 23 days ${ }^{46}$.

Recently, neural activity recorded from the dorsal horn of the spinal cord has been used to demonstrate the feasibility of estimating the pressure ${ }^{26}$ and volume ${ }^{47}$ of the bladder. For this purpose, a microelectrode array was implanted in the spinal dorsal horn of the L6 to the S1 segment. It was demonstrated that the firing rates of the sorted neurons can be used to estimate the pressure ${ }^{26}$ and volume ${ }^{47}$ during the filling of the bladder.

One important issue in decoding continuous action from neural recordings is the decoding model itself. The most common approaches used in the past to monitor the bladder have been linear regression ${ }^{26,27,36,47}$ and Kalman filtering ${ }^{27}$. Despite the existence of efficient linear decoding models, linear regression models only look at the linear relationship between the mean of the dependent variable and the independent variables, while neural signals originate from highly nonlinear and multidimensional systems. Moreover, linear approaches are relatively sensitive to outliers. To improve the performance of the decoding model, some studies used nonlinear regression methods, such as nonlinear autoregressive moving average (NARMA) models ${ }^{27}$ and support vector regression $(\mathrm{SVR})^{26} \cdot \mathrm{In}^{27}$, it was demonstrated that the NARMA model provided the most accurate bladder pressure estimate (based on the normalized root-mean-squared error) compared to Kalman filtering and linear regression. Moreover, it was shown that the decoding accuracy achieved by SVR was significantly greater than that achieved by the linear filter ${ }^{26}$. However, the nonlinear regression used $\mathrm{in}^{26}$ is a static structure in which there is no feedback, and the outputs are calculated directly based on the inputs (i.e., firing rates). The static structure of the decoding method is not an efficient method for representing the nonlinear dynamic relationships between neural signals and bladder states. Moreover, it has difficulty capturing global system behaviors in the identification of nonlinear systems that combine long- and short-term dynamics.

In this paper, we propose a deep recurrent neural network (DRNN) for the estimation of bladder pressure and volume from extracellular neural activity recorded directly from spinal cord gray matter neurons. The model is based on the Long Short-Term Memory (LSTM) architecture, which has emerged as a general and effective model for capturing long-term temporal dependencies ${ }^{48}$. The LSTM is a state-based RNN whose output depends not only on the current information to perform the estimation but also explicitly takes into account the long-term information from the past.

In addition to spiking activity, it has been demonstrated that the local field potential (LFP) activity recorded from the lumbosacral dorsal horn also carries information about intravesical pressure ${ }^{26}$. Neural information processing at each level of observation (spiking, LFP, electroencephalogram) entails the interaction of both evoked (input-driven or stimulus-related) and induced (background processes or intrinsic dynamics) factors. In this paper, we combine modeling of spiking activity and LFP activity into a unified framework to estimate the pressure and volume of the bladder.

Afferents innervating the bladder project to the lumbosacral (L6-S1) segments of the rat spinal cord. The question arises as to which segment provides more information about the bladder. To answer this question, we investigated single-electrode recordings from the L6 and S1 segments using mutual information and decoding performance.

Another important issue in the closed-loop control of the bladder and in developing an efficient therapeutic approach for individuals with spinal cord injury or neurological disorders is the simultaneous measurement of both the pressure and the volume of the bladder. To date, researchers have focused on estimating either the intravesical pressure ${ }^{26,27}$ or volume $\mathrm{e}^{36,47}$ from neural signals. In this paper, we also investigate the simultaneous estimation of both the pressure and volume of the bladder from single-electrode lumbosacral spinal recording using the proposed decoding model. 


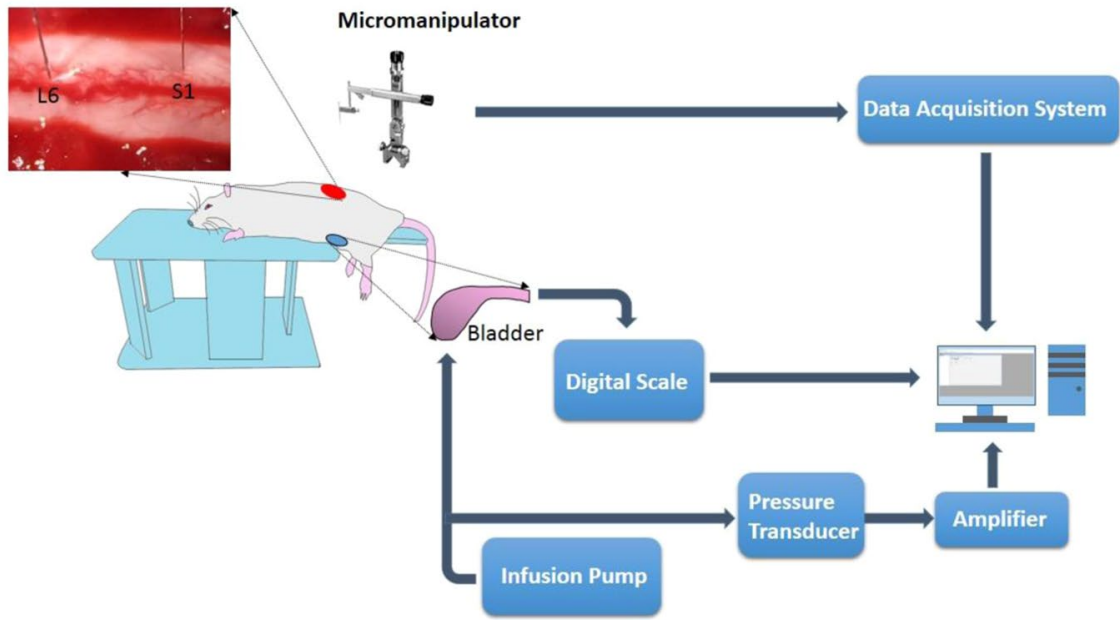

(a)

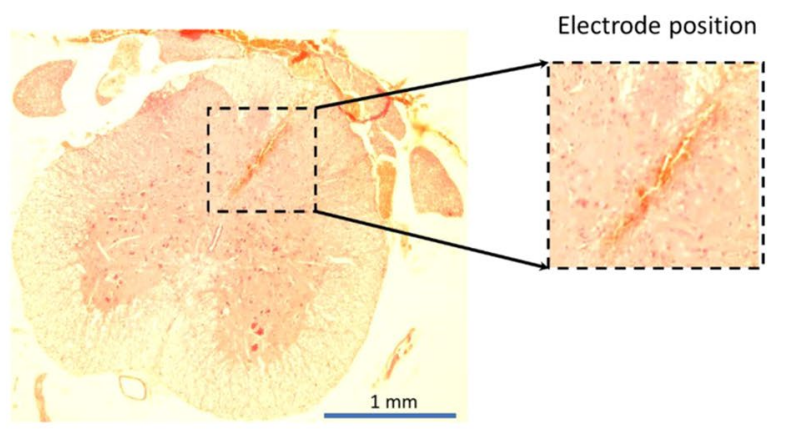

(b)

Figure 1. Experimental setup and recording sites. (a) A catheter was inserted into the bladder wall thorough the dome and secured with a purse-string suture. One head of the catheter was connected to the pressure transducer and another head to the infusion pump. A digital scale was positioned under the rat to measure the voided volume. One single-needle electrode was inserted into L6, and another one was inserted into the $\mathrm{S} 1$ spinal cord segment for neural activity recording. Bladder filling was performed with $0.9 \%$ saline at room temperature using an infusion pump at different rates $(6,8$, and $10 \mathrm{ml} / \mathrm{h})$. (b) A typical histological image from the S1 spinal cord segment of rat 8. Staining was performed with hematoxylin and eosin (H \& E).

\section{Materials and Methods}

Animal preparation and surgery. The experiments were conducted on fifteen intact adult male Wistar rats (180-400 gr). All surgical procedures and experimental protocols involving animal models described in this paper were approved by the Institutional Animal Care and Ethics Committee of Iran Neural Technology Research Center, Iran University of Science and Technology. The whole protocols and methods were performed in accordance with the recommendations and relevant guidelines for the care and use of laboratory animals. The animals were anesthetized with urethane $(1.5 \mathrm{~g} / \mathrm{kg}$, intraperitoneally) and remained sedated with ketamine $(3 \mathrm{mg} /$ $\mathrm{kg}$ ). The fur around the T13 to the L4 vertebrae was removed. Then, the skin was incised along the vertebrae, and the muscle tissue was removed until the vertebrae were visible. A laminectomy was performed on the lumbar vertebrae (L1 to L2) to expose the L6 to S1 spinal cord segments. To expose the bladder, a ventral midline incision was made. A sterile polyethylene (PE) 50 tube $(0.5 \mathrm{~mm}$ ID and $0.9 \mathrm{~mm}$ OD) (AD Instruments Ltd, Australia) was inserted into the bladder wall through the dome and secured with a purse-string suture. The PE tube was attached to a stopcock connected to a pressure transducer (NovaTrans transducer system, MX860, Smiths Medical ASD, Inc.) and a syringe pump (SN-50C6, Sino Medical-Device Technology Co., Ltd., China) for recording the intravesical bladder pressure and infusing saline into the bladder, respectively. The pressure signals were amplified $(900 \times)$ and sampled at $50 \mathrm{~Hz}$ (maximum sampling rate of pressure sensor is $1 \mathrm{kHz}$ ). A digital scale (GF-300, $\mathrm{A} \& \mathrm{D}$ Instruments Ltd., UK) was positioned under the rat to measure the voided volume. The volume signals were sampled at $5 \mathrm{~Hz}$. The experimental setup is illustrated in Fig. 1. The bladder was filled with three different rates of saline solution (i.e., 6,8 , and $10 \mathrm{ml} / \mathrm{h}$ ), and the neural signals and the pressure and volume of the bladder were recorded simultaneously during filling. Each trial started with the saline infusion and continued until several voiding contractions and bladder leakages occurred. The duration of each trial was between 450 and 850 seconds. At the end of each trial, the bladder was emptied with a syringe, and the rat was relaxed for 20 minutes. During 


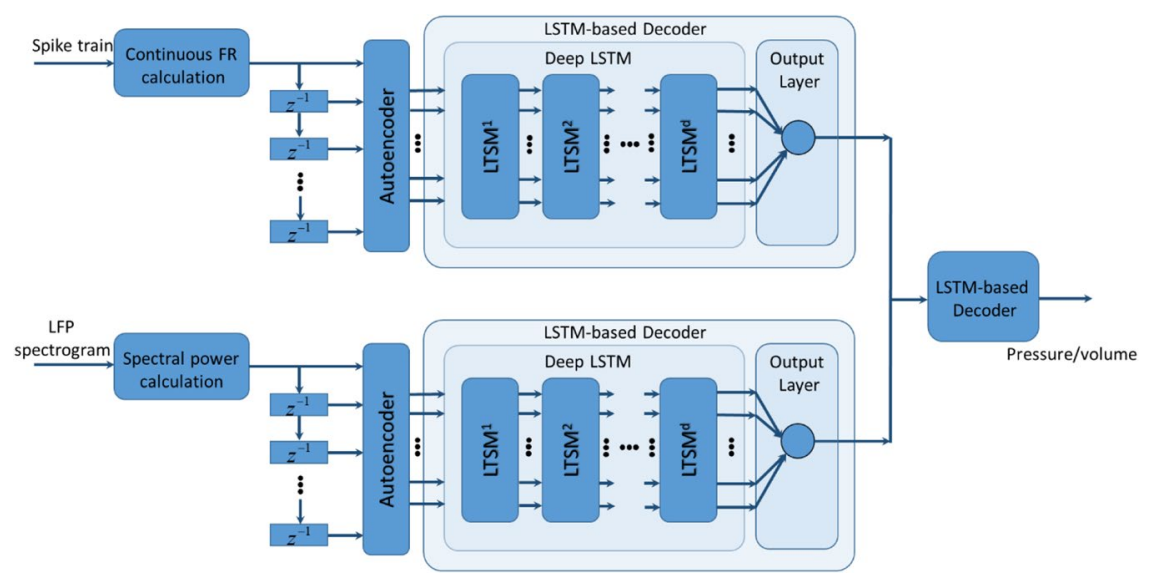

Figure 2. The architecture of the proposed decoding model. The model is based on an autoencoder (AE) and a recurrent neural network with long Short-Term Memory (LSTM).

single-electrode recordings, each session of the experiment consisted of four trials per rat. During two-electrode recordings, 2-3 trials of the experiment were conducted per rat. In total, 53 trials were conducted on 15 rats.

Electrode implantation and neural signal acquisition. Recording electrodes were made from epoxylite-insulated tungsten with shank diameter $75 \mu \mathrm{m}, 5^{\circ}-10^{\circ}$ tapered tip of $120 \mu \mathrm{m}$ exposed length, and $300-500 \mathrm{k} \Omega$ resistance (FHC Inc., Bowdin, ME USA). The microelectrodes were mounted on a micromanipulator (SM-15, Narishige Group Product, Japan) that could control the three-dimensional positioning of the electrode with a minimum graduation of $10 \mu \mathrm{m}$. The electrodes were positioned at locations within the L6 and S1 dorsal horn approximately $0-1 \mathrm{~mm}$ lateral from the midline between 100 and $200 \mu \mathrm{m}$ in depth (Fig. 1(b)). To determine the best electrode position within the dorsal horn, the electrode was vertically advanced through the spinal cord dorsoventrally. Then, the electrode was withdrawn and moved $100 \mu \mathrm{m}$ mediolaterally and/or rostrocaudally to an adjacent location, while the correlation of neural activity with the bladder pressure was visually inspected on the monitor of the recording system. The positions that produced the highest correlations were selected. The recording process was performed within a custom-made Faraday cage to increase the signal-to-noise ratio. Neural signals were amplified (programmable gain, $1000 \times$ ) and recorded at a $20 \mathrm{kHz}$ sampling rate using a data acquisition system (USB-ME64 system, Multichannel Systems Reutlingen, Germany).

Preprocessing and feature extraction. The recorded neural signals were bandpass filtered between 300 and $3000 \mathrm{~Hz}$ with the low-pass and high-pass elliptic filters of order four. Spikes were detected using the Wave_ Clus program, which is publicly available online (http://www2.le.ac.uk/centres/csn/research-2/spike-sorting). The threshold for spike detection was set to four times the standard deviation of the noise estimated from the filtered signal, and spike events were identified as each instance the signal exceeded this threshold. The feature set was formed from the continuous firing rate (FR) and the LFP. All data analyses and decoding models were performed with customized algorithms written in MATLAB.

Continuous FR. Continuous FR was computed by taking a Gaussian window of duration 50 ms and counting the number of spikes within the window at each time. Then, the FR was smoothed using a causal moving average filter with a span of 20 samples.

Local field potential. The power of the LFP subband component constituted the second feature set. The LFP signals were down sampled to $2000 \mathrm{~Hz}$. Short-time Fourier transform (STFT) was used to generate power spectra over time with a $500 \mathrm{~ms}$ Hanning window with $80 \%$ overlap. The average of the power spectrum in five frequency bands $(1-2.9 \mathrm{~Hz}, 3-8.8 \mathrm{~Hz}, 9.1-26.7 \mathrm{~Hz}, 27.7-81 \mathrm{~Hz}, 83.9-256 \mathrm{~Hz})$ was used as the neural signal feature for decoding the pressure and the volume of the bladder. The average of the power spectrum was smoothed using a moving average filter with a span of 10 samples. The frequency bands of the LFP signals were selected according to ${ }^{26}$.

Decoding model. The architecture of the proposed decoding model is shown in Fig. 2. The model is based on an autoencoder $(\mathrm{AE})^{49}$ and a recurrent neural network with LSTM $^{50,51}$. Autoencoder is one of the deep architecture-based models used to learn low-dimensional features from a high-dimensional input vector ${ }^{49}$. LSTM is a type of RNN that allows learning of long-term temporal dependencies. In principle, the RNN involves dynamic elements in the form of a feedback loop, which feedback the lagged outputs of the neurons to the inputs of neurons ${ }^{52}$. The feedback loops enable the network to perform dynamic mapping and learn tasks that extend over time. However, conventional RNN encounters challenges in modeling long-term dependencies, such as vanishing and exploding gradient problems ${ }^{48,53}$. Recurrent neural networks with LSTM have been shown to be an efficient method to overcome these problems. 


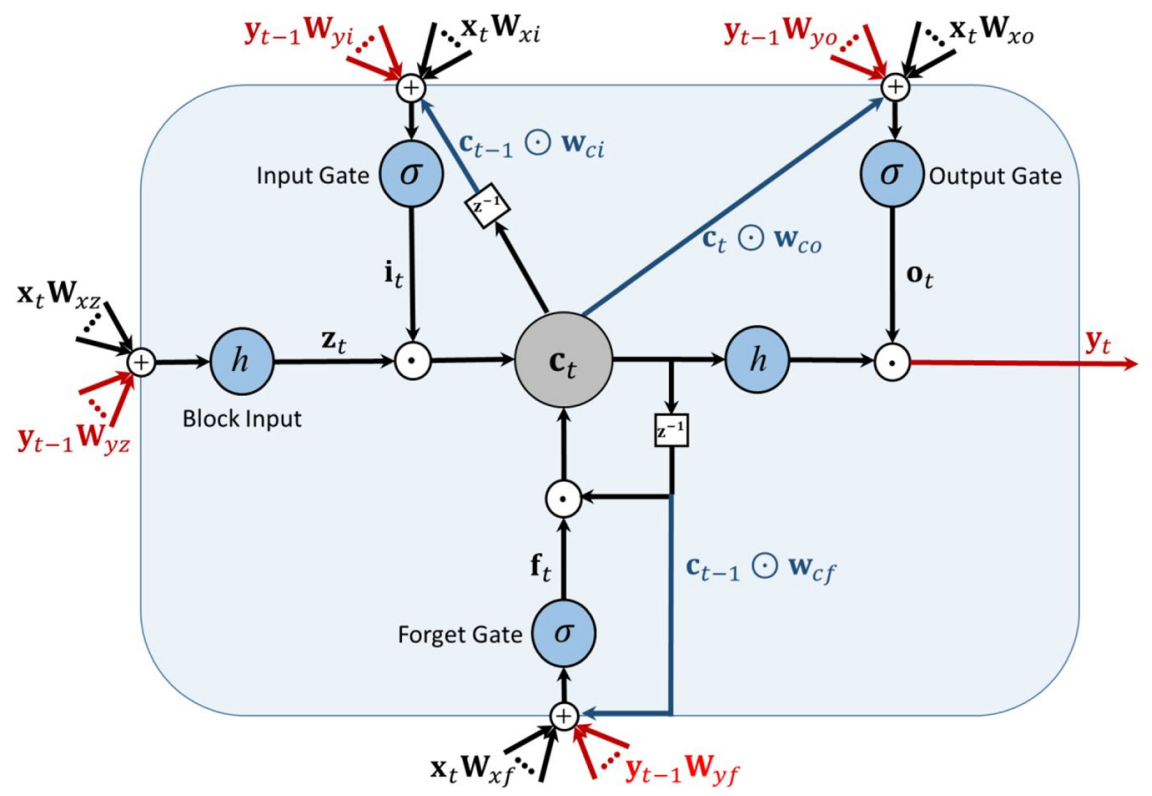

Figure 3. Detailed schematic of a Long Short-Term Memory block (LSTM).

By taking advantage of an AE to extract the effective features and an RNN with LSTM to take into account the long-term information in the past, a deep learning structure is proposed for estimating the pressure and volume of the bladder.

Autoencoder. The basic architecture of an AE network consists of three layers, one input layer, one hidden layer with dimensions less than the dimensions of the input layer, and an output layer with dimensions equal to the dimensions of the input layer ${ }^{49}$. The AE network transforms the high-dimensional input data into a feature space with lower dimensions, while the decoder network can reconstruct the input data from the feature space. The $\mathrm{AE}$ attempts to make the network output value close to the input value by minimizing the reconstruction error. After training the $\mathrm{AE}$, the output layer together with its connections from the hidden layer are removed, and the hidden layer is considered the extracted feature. The number of neurons in the input layer and hidden layer are 41 and 30, respectively.

Deep LSTM. A schematic diagram of the RNN with LSTM is shown in Fig. 3. Each LSTM layer consists of three gates (input, output, forget), block input, block output, memory cell, and peephole connections. The output of the LSTM layer is recurrently connected back to the input layer and to all of the gates of the LSTM layer. The input gate determines the amount of new information entered into the block, the forget gate determines when to forget content regarding the internal state, and the output gate controls the amount of information going to the output. The input, forget, and output gates have a sigmoid activation function, $\sigma$, and a hyperbolic tangent, $h$, is usually selected as the activation function of the block input and output. We may then describe the dynamic behavior of the network by the following equations:

$$
\begin{gathered}
\mathbf{i}_{t}=\sigma\left(\mathbf{x}_{t} \mathbf{W}_{x i}+\mathbf{y}_{t-1} \mathbf{W}_{y i}+\mathbf{w}_{c i} \odot \mathbf{c}_{t-1}+\mathbf{b}_{i}\right) \\
\mathbf{f}_{t}=\sigma\left(\mathbf{x}_{t} \mathbf{W}_{x f}+\mathbf{y}_{t-1} \mathbf{W}_{y f}+\mathbf{w}_{c f} \odot \mathbf{c}_{t-1}+\mathbf{b}_{f}\right) \\
\mathbf{z}_{t}=h\left(\mathbf{x}_{t} \mathbf{W}_{x z}+\mathbf{y}_{t-1} \mathbf{W}_{y z}+\mathbf{b}_{z}\right) \\
\mathbf{c}_{t}=\mathbf{f}_{t} \odot \mathbf{c}_{t-1}+\mathbf{i}_{t} \odot \mathbf{z}_{t} \\
\mathbf{o}_{t}=\sigma\left(\mathbf{x}_{t} \mathbf{W}_{x o}+\mathbf{y}_{t-1} \mathbf{W}_{y o}+\mathbf{w}_{c o} \odot \mathbf{c}_{t}+\mathbf{b}_{o}\right) \\
\mathbf{y}_{t}=\mathbf{o}_{t} \odot h\left(\mathbf{c}_{t}\right)
\end{gathered}
$$

where $\mathbf{x}$ is the input vector to the LSTM, $\mathbf{W}_{x i}, \mathbf{W}_{x z}, \mathbf{W}_{x f}$, and $\mathbf{W}_{x o}$ are the connection weights from the input to the input gate, block input, forget gate, and output gate, respectively, $\mathbf{W}_{y i}, \mathbf{W}_{y z}, \mathbf{W}_{y f}$, and $\mathbf{W}_{y o}$ are the recurrent connection weights from the output to the input gate, block input, forget gate, and output gate, respectively, and $\mathbf{w}_{c i}, \mathbf{w}_{c f}$, and $\mathbf{w}_{c o}$ are the peephole connection weights from the cell gate to the input gate, forget gate, and output gate, 
respectively. Vectors $\mathbf{b}_{i}, \mathbf{b}_{f}, \mathbf{b}_{z}$, and $\mathbf{b}_{o}$ are the bias weights determined during training. Symbol $\odot$ represents pointwise multiplication. Three LSTM layers are considered and each LSTM layer contains 10 units. The number of units and the number of LSTM layers are selected heuristically to achieve the best performance.

The AE and LSTM-based decoder was trained using the Levenberg-Marquardt and stochastic gradient descent method, respectively. The Levenberg-Marquardt method is a compromise between the Gradient descent, which has a guaranteed convergence upon a proper choice of the step-size, and Newton's method, which converges speedily near a local or global minimum.

Data analysis methods. To assess the performance of the proposed method in estimating bladder pressure and volume, the normalized root-mean-square (NRMS) of the estimation error and the correlation coefficient (CC), were used. The NRMS and CC were defined as

$$
\begin{gathered}
\operatorname{NRMS}(\%)=\frac{1}{\max \left(y_{d}(t)\right)-\min \left(y_{d}(t)\right)} \times \sqrt{\frac{1}{T} \sum_{t=1}^{T}\left(y_{d}(t)-y(t)\right)^{2}} \times 100 \\
\mathrm{CC}(\%)=\left(\frac{\sum_{t=1}^{T}(y(t)-\bar{y})\left(y_{d}(t)-\bar{y}_{d}\right)}{\sum_{t=1}^{T}(y(t)-\bar{y})^{2} \sum_{t=1}^{T}\left(y_{d}(t)-\bar{y}_{d}\right)^{2}}\right) \times 100
\end{gathered}
$$

where $y_{d}(t)$ is the desired pressure or volume and $y(\mathrm{t})$ is the estimated value of desired pressure or volume. Moreover, to evaluate the information content of the neural signals recorded from different vertebral segments, the mutual information (MI) was calculated between the neural signal feature of interest (firing rate or LFP signal band power spectrum) of each vertebral segment and the bladder parameter of interest (pressure or volume). Mutual information was calculated based on an adaptive partitioning of the observation space ${ }^{54}$. Two-way analysis of variance (ANOVA) was used to assess the statistical significance of the results and differences, and a confidence level of $95 \%(p<0.05)$ was chosen to indicate a significant difference.

Three methods were used for evaluating the proposed method: inside-trial, trial-by-trial, and rat-by-rat. For the inside-trial evaluation, the deep LSTM was trained and tested with the data obtained during each trial of experiment. In this case, $70 \%$ of the data were used for training, and the remaining $30 \%$ of the data were used for testing. For the trial-by-trial evaluation, training was performed on the data obtained during one trial of the experiment and tested with the remaining trials. For the rat-by-rat evaluation method, the deep LSTM was trained with one trial of the experiments on one rat and tested with the data obtained from each rat. In total, 53 trials were conducted on 15 rats. The duration of each trial was between 450 and 850 seconds.

\section{Results}

Figure 4 shows the recorded infused volume, residual volume, voided volume, bladder pressure, bandpass filtered neural signal $(0.3-3 \mathrm{kHz})$, continuous firing rate, intraspinal LFP activity and corresponding time-frequency analysis during a typical trial from the experiment (rat 5 , trial 3 , infusion rate $=10 \mathrm{ml} / \mathrm{h}$ ). It is observed that both the bladder pressure and the firing rate monotonically increase with increasing bladder volume. When the bladder pressure suddenly increases, bladder leakage occurs.

The time-frequency analysis of the intraspinal LFP shows that spectral patterns could clearly reflect the status of the bladder. Figure 5 shows the typical frequency bands of the intraspinal LFP signal during bladder filling. The power of the defined frequency bands suddenly increases as the bladder pressure increases, and the local maxima of the LFP frequency bands show the instant when bladder leakage occurs.

To assess the information content in the frequency bands of intraspinal LFP signals and in the continuous firing rate with respect to bladder pressure and volume, the averages of the $\mathrm{CC}$ and mutual information for 40 trials of experiment on 10 rats were computed (Fig. 6).

The results show that the firing rate provides more information about bladder pressure and volume $(p<0.05$ based on both CC and MI. The comparison was performed between the mutual information and CC obtained from both the frequency bands and the FR. It can be seen that the neural signals recorded from S1 contain significantly more information about bladder pressure than those recorded from L6 $(p=0.013$ for CC and $p=0.01$ for MI) but that there was no significant difference in the information between S1 and L6 with respect to volume ( $p=0.0836$ for MI and $p=0.429$ for CC). Moreover, the results of the statistical test show that the high frequency components of the LFP signals provide a higher correlation and greater information than the low frequency components $(p<0.05)$ with respect to both bladder pressure and volume. Comparisons were performed between low frequency bands (FB1, FB2, FB3, and FB4) and high frequency bands (FB5) using CC as well as MI.

Single electrode decoding. In this section, the performance in decoding bladder pressure/volume using signals recorded from the L6 segment and the S1 segment are presented. Figure 7 shows a typical decoding of the pressure/volume during one trial of the experiment (rat 5, trial 3, infusion rate $=10 \mathrm{ml} / \mathrm{h}$ ) using FR, LFP band power spectra, and a combination of FR and LFP band power spectra. It is observed that excellent decoding performance is obtained using the combination of FR and LFP band power spectra. The decoding errors are 7.7\% and $15.4 \%$ for pressure and volume, respectively.

Table 1 summarizes the average inside-trial decoding performance obtained using the neural signals recorded from the L6 segment and the S1 segment for 40 trials of experiments on 10 rats. For both pressure and volume estimation, the decoding performance obtained using the combined FR and LFP data was significantly better than that obtained using FR or LFP alone ( $p<0.001$ for both NRMS and CC). Moreover, the CC shows that the S1 signals provided significantly better decoding performance than those of the L6 $(p<0.0013)$. 
(a)

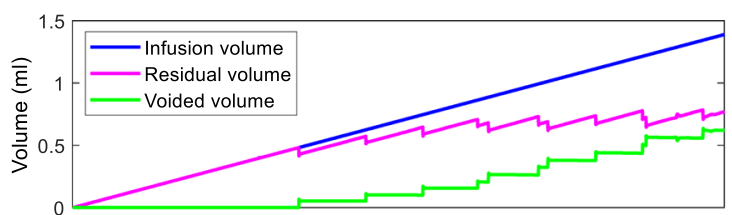

(b)
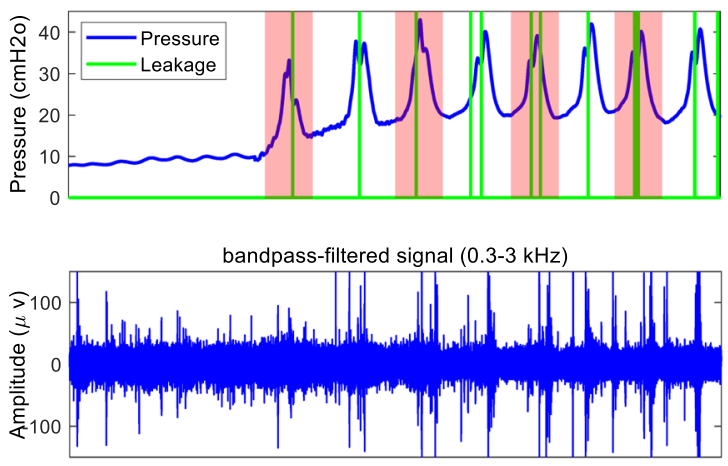

(d)
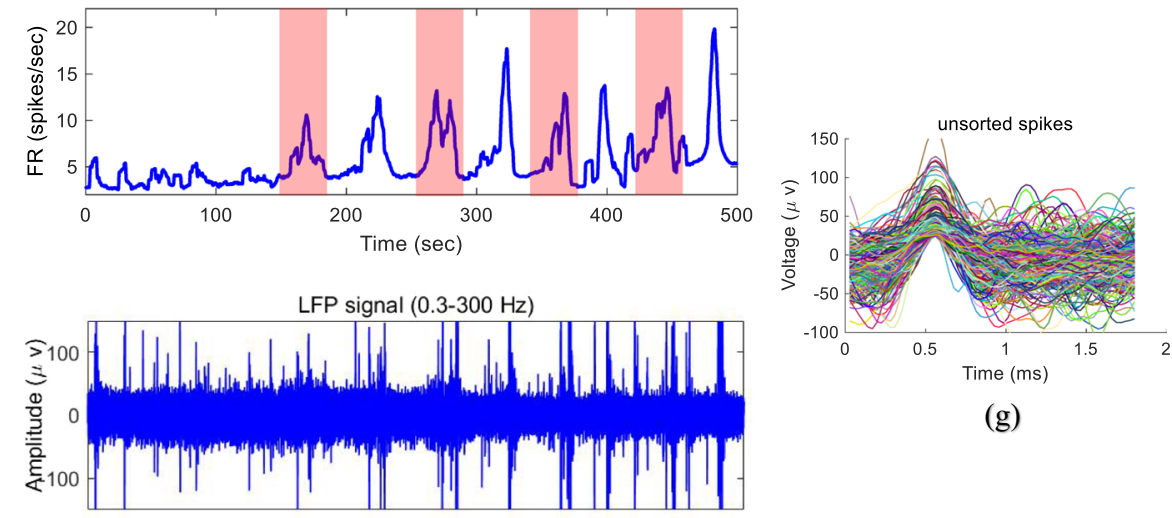

(e)

(g)

(f)

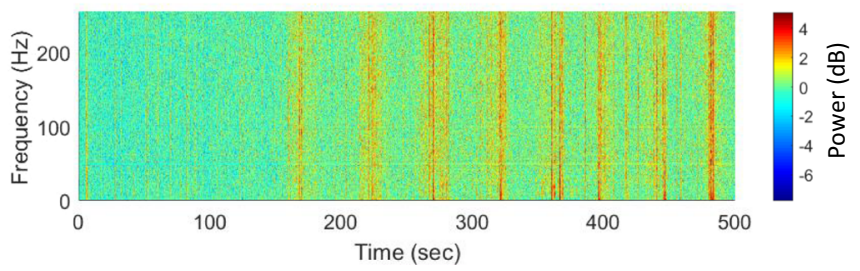

Figure 4. Typical signals recorded from the bladder (rat 5 , trial 3 , infusion rate $=10 \mathrm{ml} / \mathrm{h}$ ). (a) Infusion volume, residual volume, and voided volume. (b) Bladder pressure and leakage events. (c) Bandpass filtered $(0.3-3 \mathrm{kHz})$ neural signal from S1. (d) Continuous firing rate of the detected spikes. (e) Recorded local field potential (LFP) signal. (f) Time-frequency analysis of the LFP signal. (g) Unsorted detected spike waveforms.

Figure 8(a) shows the result of a typical decoding of pressure and volume using the trial-by-trial validation approach. Training was performed with rat 5 , trial 1 with an infusion rate of $8 \mathrm{ml} / \mathrm{h}$, and testing was performed on the same rat, trial 3 with an infusion rate of $10 \mathrm{ml} / \mathrm{h}$. The NRMS decoding errors for pressure decoding were $19.6 \%, 12.6 \%$, and $7.6 \%$ using LFP, FR, and both LFP and FR, respectively, and $30.8 \%, 17.3 \%$, and $10.9 \%$, respectively, for volume decoding. The CC values for pressure decoding were $87.2 \%, 90.1 \%$, and $97.8 \%$ using LFP, FR, and combined LFP and FR, respectively, and 95.2\%, 84.0\%, and 97.5\%, respectively, for volume decoding. The results indicate that the combination of LFP and FR provided better performance than using only LFP or FR in terms of both NRMS and CC indices. The decoding performance from single-electrode recording from the S1 segments of 10 rats using trial-by-trial validation are summarized in Table 2 . The average pressure decoding errors were $26.5 \pm 5.0 \%, 22.3 \pm 5.2 \%$, and $17.8 \pm 4.8 \%$ using LFP, FR, and the combination of LFP and FR, respectively, and $30.0 \pm 6.3 \%, 25.4 \pm 3.5 \%$, and $21.3 \pm 4.5 \%$, respectively, for volume decoding. Additionally, the average CCs for pressure decoding were $66.0 \pm 8.0 \%, 74.2 \pm 6.5 \%, 80.3 \pm 7.3 \%$ using LFP, FR, and the combination of LFP and FR, respectively, and $60.9 \pm 13.1 \%, 65.3 \pm 7.8 \%$, and $70.5 \pm 9.9 \%$, respectively, for volume decoding. The results for pressure decoding show that the combined LFP and FR provided significantly better performance than LFP or FR alone ( $p<0.001$ for NRMS and CC). The results show that the decoding error obtained for volume using the combined LFP and FR was significantly less than that obtained using only LFP or FR ( $p=0.0011$ for NRMS). Moreover, the CC shows that the decoding performance obtained using the combined LFP and FR was better than that obtained using only LFP or FR, but the difference was not statistically significant $(p=0.1169)$. 
(a)

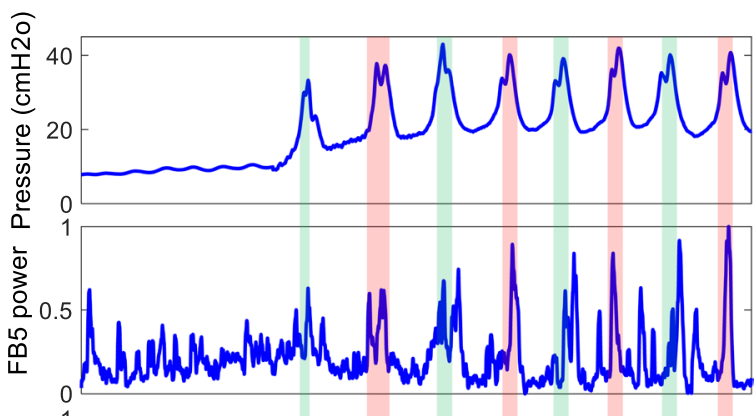

(c)

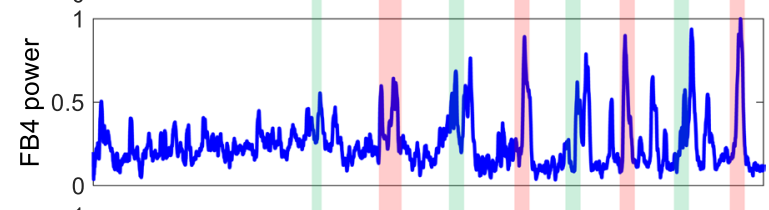

(d)

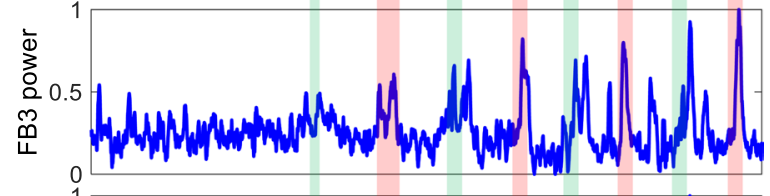

(e)

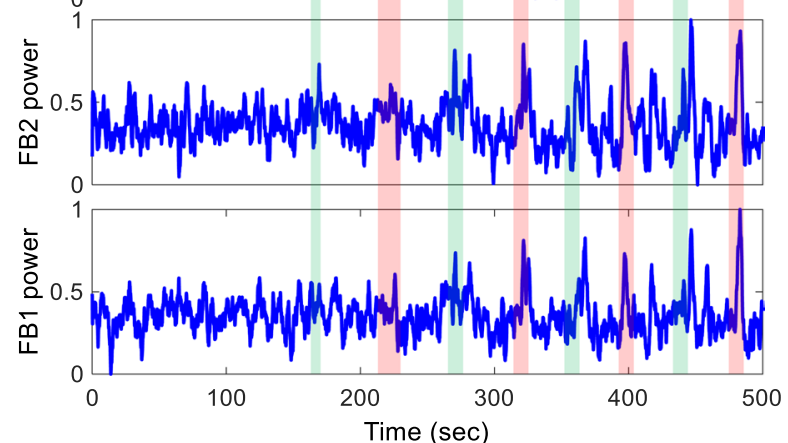

Figure 5. An example of the bladder pressure and power spectrum of the recorded LFP signal recorded from $\mathrm{S} 1$ (trial 3, rat 5, infusion rate $=10 \mathrm{ml} / \mathrm{h}$ ) in different frequency bands: FB5: 83.9-256 Hz, FB4: $27.7-81 \mathrm{~Hz}$, FB3: 9.1-26.7 Hz, FB2: 3-8.8 Hz, FB1: 1-2.9 Hz. Colored vertical bars are provided to help visualize changes in frequency bands across the subplots as the bladder pressure changes.

Figure 8 (b) shows an example of decoding using rat-by-rat validation. Training was performed on rat 7 , trial 1 , with an infusion rate of $8 \mathrm{ml} / \mathrm{h}$, and testing was performed on rat 5 , trial 3 with an infusion rate of $10 \mathrm{ml} / \mathrm{h}$. The NRMS decoding errors obtained were $22.4 \%, 21.7 \%$, and $14.1 \%$ using LFP, FR, and both LFP and FR, respectively, and $20.0 \%, 25.1 \%$, and $18.9 \%$, respectively, for volume decoding. The CC values for pressure decoding were $93.2 \%, 60.0 \%$, and $96.3 \%$ using LFP, FR, and combined LFP and FR, respectively, and $97.3 \%, 83.7 \%$, and $96.8 \%$, respectively, for volume decoding. The results show that the decoding model is robust with respect to the training rat. The results of the decoding performance using the rat-by-rat validation approach are summarized in Table 3. In this approach, the data obtained during one trial from one rat were used to train the model, and the data obtained during all trials from all rats were used to test the model. The results indicate that using the combined FR and LFP provides significantly better performance than using only FR or LFP for both pressure and volume ( $p=0.0033$ for pressure and $p=0.0015$ for volume using NRMS). The results show that the training data obtained from different rats have no significant effect on the decoding performance $(p=0.3140$ for pressure and $p=0.2596$ for volume using NRMS).

Two-electrode decoding. In two-electrode decoding, two electrodes were implanted into the S1 and L6 spinal cord segments, and the compound signals recorded from both segments were used for decoding. Figure 9 shows a typical pressure and volume decoding using the signal recorded from S1, L6, or the combined signal from S1 and L6 during two-electrode recording. Figure 9(a) illustrates the inside-trial decoding results. The NRMS values obtained for pressure decoding were $15.4 \%, 17.1 \%$, and $6.3 \%$ using S1, L6, and the combined signals from S1 and L6, respectively, and $20.0 \%, 28.1 \%$, and $14.2 \%$, respectively, for volume decoding. The CC values for pressure decoding were $79.6 \%, 73.0 \%$, and $96.8 \%$ using S1, L6, and the combined signals from L6 and S1, respectively, and $78.9 \%, 50.4 \%$, and $83.9 \%$, respectively, for volume decoding. Figure 9 (b) shows the trial-by-trial decoding results. The NRMS pressure decoding errors obtained were $12.5 \%, 16.7 \%$ and $6.6 \%$ using S1, L6, and the combined signals recorded from S1 and L6, respectively. Compared to the single-electrode decoding, the two-electrode decoding yielded $47.2 \%$ and $60.5 \%$ improvement using S1 and L6, respectively. The NRMS volume decoding errors obtained were $15.3 \%, 16.2 \%$ and $8.7 \%$ using the signals recorded from S1, L6, and the combined signal 


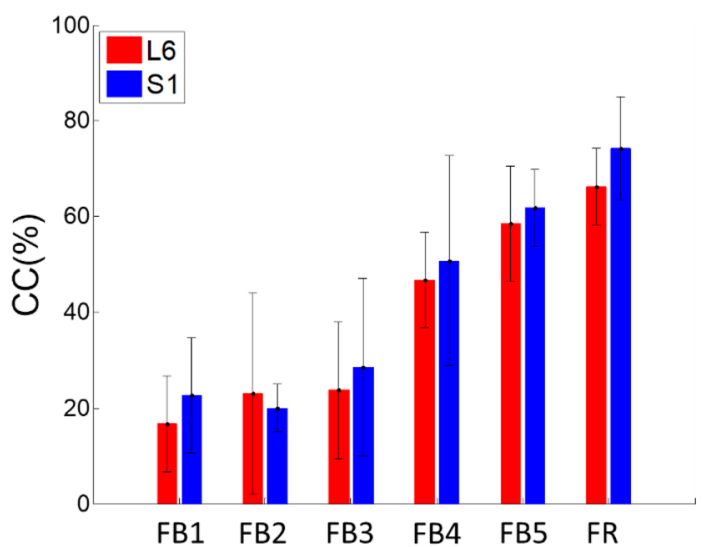

(a)

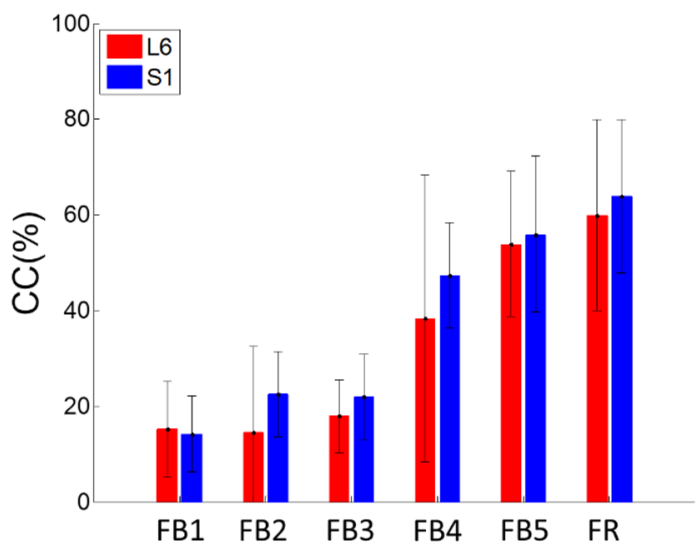

(b)

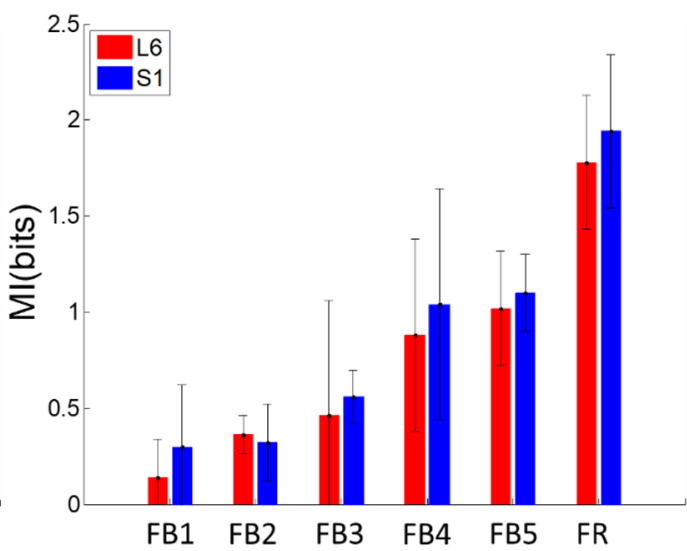

(c)

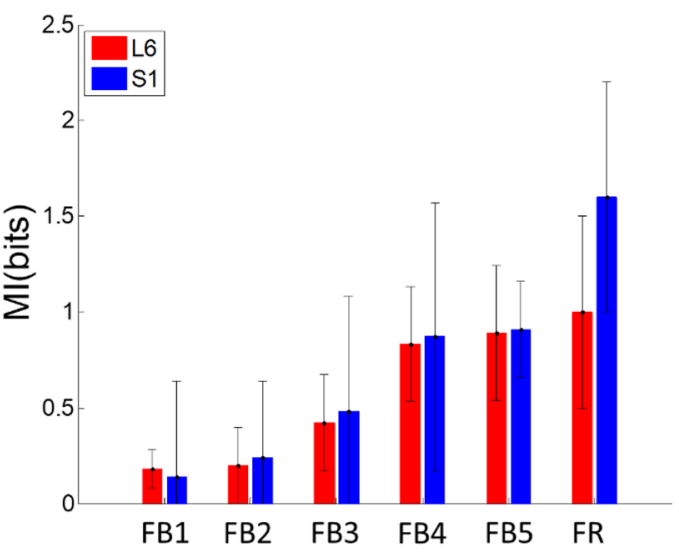

(d)

Figure 6. Correlation coefficient and mutual information between bladder statuses with extracted features. (a) Correlation coefficient between bladder pressure and extracted features. (b) Correlation coefficient between volume and extracted features. (c) Mutual information between pressure and extracted features. (d) Mutual information between volume and extracted features. Error bars indicate standard deviations.

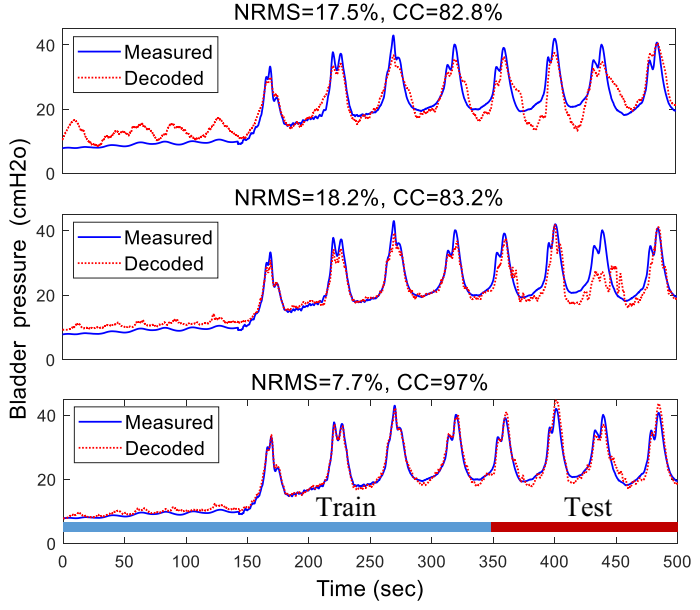

(a)

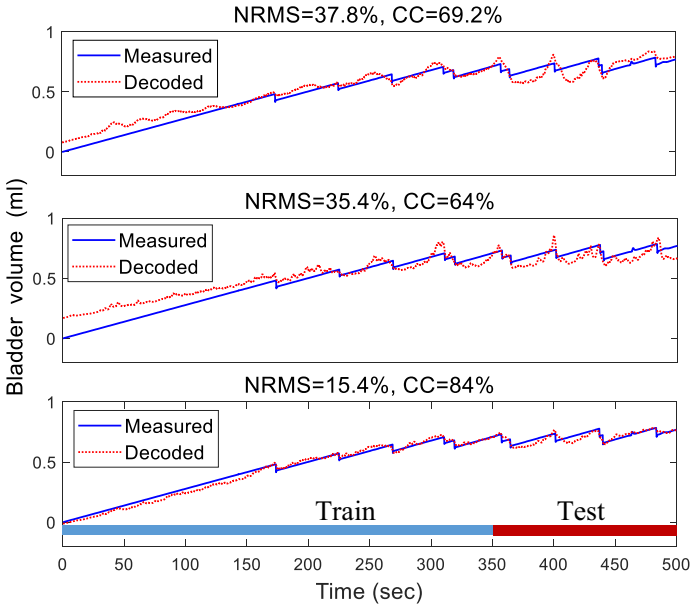

(b)

Figure 7. An example of decoding the bladder pressure (a) and volume (b) using an inside-trial validation approach (training with $70 \%$ of trial 3 and testing with the remaining $30 \%$ of the same trial) on rat 5 (infusion rate $=10 \mathrm{ml} / \mathrm{h}$ ). Decoding was performed using the signal recorded from $\mathrm{S} 1$ for different extracted feature sets: firing rate (upper trace), FB5 subband power spectrum of the LFP (middle trace), and the combined FR and FB5 subband power spectrum (lower trace). The NRMS and CC values given refer to the test period. 


\begin{tabular}{|l|l|l|l|l|l|}
\hline \multicolumn{2}{|c|}{} & \multicolumn{3}{|l|}{ S1 segment } & L6 segment \\
\cline { 3 - 7 } \multicolumn{2}{|c|}{} & NRMS\% & CC\% & NRMS\% & CC\% \\
\hline \multirow{2}{*}{ FR } & pressure & $22.5 \pm 4.8$ & $72.5 \pm 12.4$ & $23.6 \pm 5.1$ & $69.7 \pm 8.1$ \\
\cline { 2 - 6 } & volume & $28.2 \pm 6.9$ & $60.8 \pm 13.4$ & $30.2 \pm 4.7$ & $56.3 \pm 10.3$ \\
\hline \multirow{2}{*}{ LFP } & pressure & $24.6 \pm 8.9$ & $66.6 \pm 14.2$ & $26.8 \pm 5.0$ & $64.0 \pm 13.3$ \\
\cline { 2 - 6 } & volume & $32.4 \pm 5.1$ & $55.2 \pm 12.8$ & $35.6 \pm 5.3$ & $48.4 \pm 12.0$ \\
\hline \multirow{2}{*}{ FR \& LFP } & pressure & $18.3 \pm 6.7$ & $78.2 \pm 18.2$ & $20.7 \pm 9.4$ & $75.0 \pm 12.4$ \\
\cline { 2 - 6 } & volume & $24.2 \pm 10.9$ & $68.2 \pm 17.0$ & $27.8 \pm 5.4$ & $62.2 \pm 10.0$ \\
\hline
\end{tabular}

Table 1. Average and standard deviation of decoding performance across 10 rats using the inside-trial validation approach using single-electrode recording from S1 and L6 segments.
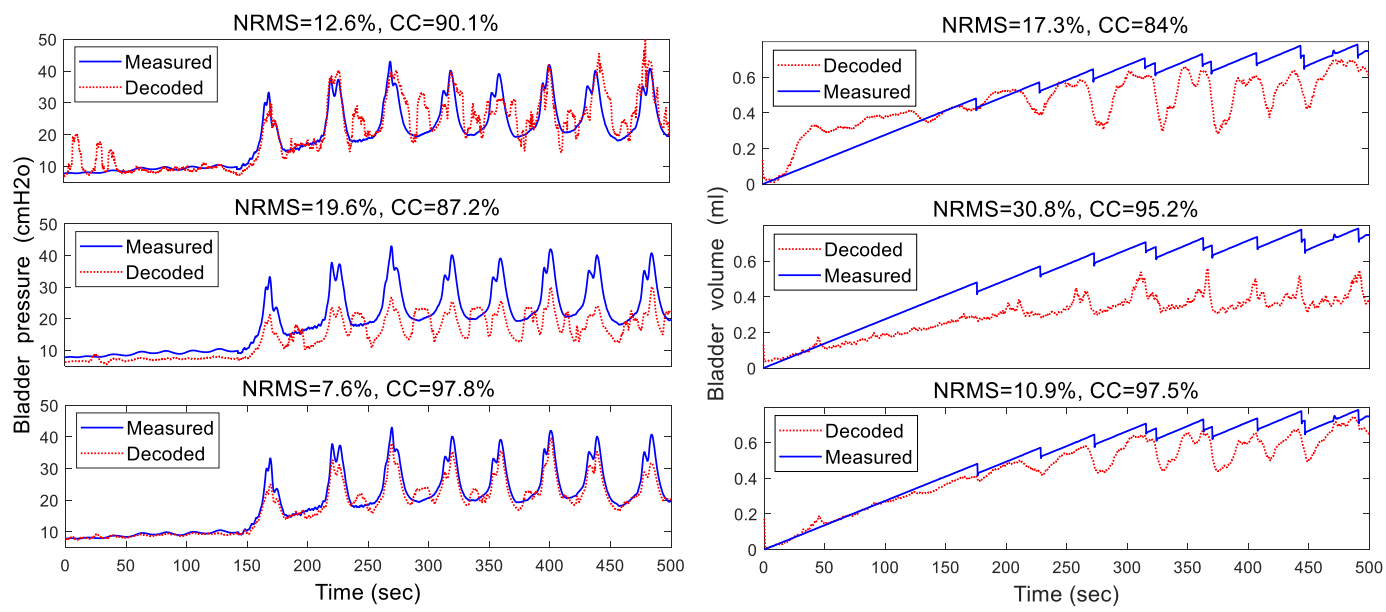

(a)
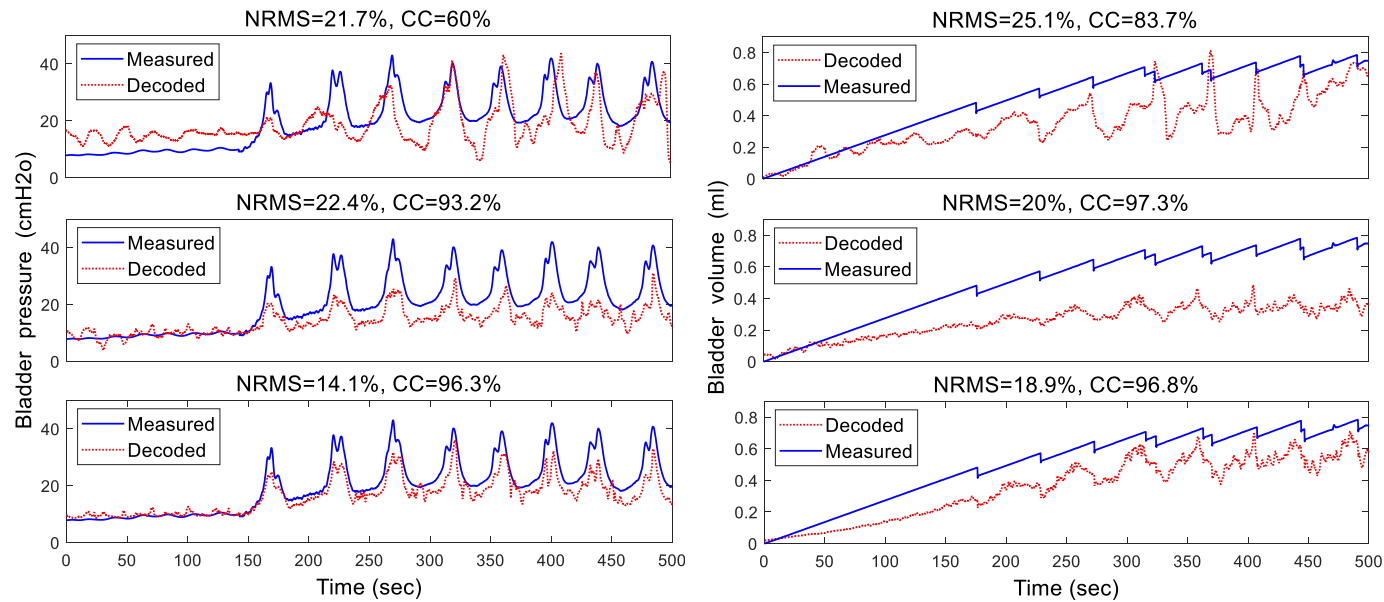

(b)

Figure 8. An example of decoding the bladder pressure (left) and volume (right) using trial-by-trial (a) and rat-by-rat (b) validation approaches. Decoding was performed using different extracted feature sets: firing rate (upper trace), FB5 subband power spectrum of the LFP (middle trace), and the combination of FR and FB5 subband power spectrum (lower trace). In this example, during the trial-by-trial validation approach, the network was trained with trial 1 from rat 5 (infusion rate $=8 \mathrm{ml} / \mathrm{h}$ ) and tested with trial 3 from the same rat (infusion rate $=10 \mathrm{ml} / \mathrm{h}$ ). During the rat-by-rat validation approach, the network was trained with rat 7 (trial 1 , infusion rate $=8 \mathrm{ml} / \mathrm{h}$ ) and tested with rat 5 (trial 3 , infusion rate $=10 \mathrm{ml} / \mathrm{h}$ ).

from S1 and L6, respectively. Using two-electrode decoding, an improvement of $43.1 \%$ and $46.3 \%$ was achieved when compared with single-electrode decoding using the signals from S1 and L6, respectively.

Table 4 summarizes the results of decoding performance for five rats during single-electrode and two-electrode decoding using the combined LFP and FR signals. In this analysis, the trial-by-trial validation approach was used. 


\begin{tabular}{|l|l|l|l|l|l|l|}
\hline \multirow{2}{*}{} & \multicolumn{3}{|l}{ LFP } & \multicolumn{2}{l|}{ FR } & \multicolumn{2}{l|}{ LFP \& FR } \\
\cline { 2 - 7 } & pressure & volume & pressure & volume & pressure & volume \\
\hline NRMS\% & $26.5 \pm 5.0$ & $30 \pm 6.3$ & $22.3 \pm 5.2$ & $25.4 \pm 3.5$ & $17.8 \pm 4.8$ & $21.3 \pm 4.5$ \\
\hline CC\% & $66.0 \pm 8.0$ & $60.9 \pm 13.1$ & $74.2 \pm 6.5$ & $65.3 \pm 7.8$ & $80.3 \pm 7.3$ & $70.5 \pm 9.9$ \\
\hline
\end{tabular}

Table 2. Average (mean \pm standard deviation) of the decoding performance across 10 rats with singleelectrode recording from S1 using the trial-by-trial validation approach.

\begin{tabular}{|c|c|c|c|c|c|c|c|c|c|}
\hline & & \multicolumn{2}{|l|}{ Train Rat 1} & \multicolumn{2}{|l|}{ Train Rat 3} & \multicolumn{2}{|l|}{ Train Rat 5} & \multicolumn{2}{|l|}{ Train Rat 7} \\
\hline & & NRMS (\%) & CC (\%) & NRMS (\%) & CC (\%) & NRMS (\%) & CC (\%) & NRMS (\%) & CC (\%) \\
\hline \multirow{3}{*}{ Pressure } & LFP & $26.2 \pm 9.2$ & $62.4 \pm 10.5$ & $28.9 \pm 6.9$ & $58.9 \pm 5.4$ & $26.3 \pm 8.4$ & $63.4 \pm 6.5$ & $27.6 \pm 9.1$ & $61.0 \pm 13.9$ \\
\hline & FR & $23.5 \pm 8.1$ & $66.3 \pm 9.6$ & $26.7 \pm 7.2$ & $62.5 \pm 9.6$ & $22.9 \pm 9.0$ & $69.2 \pm 9.9$ & $25.9 \pm 7.3$ & $65.3 \pm 12.5$ \\
\hline & FR\&LFP & $21.0 \pm 4.7$ & $71.7 \pm 8.3$ & $23.0 \pm 9.4$ & $68.8 \pm 7.4$ & $20.2 \pm 5.1$ & $73.0 \pm 7.9$ & $22.4 \pm 6.9$ & $70.0 \pm 10.3$ \\
\hline \multirow{3}{*}{ Volume } & LFP & $29.5 \pm 6.4$ & $56.7 \pm 13.4$ & $30.2 \pm 9.8$ & $54.5 \pm 9.2$ & $28.2 \pm 8.8$ & $57.3 \pm 6.7$ & $31.0 \pm 5.7$ & $54.5 \pm 15.5$ \\
\hline & FR & $26.9 \pm 6.7$ & $60.3 \pm 10.8$ & $28.2 \pm 8.2$ & $57.8 \pm 8.3$ & $24.8 \pm 9.7$ & $62.2 \pm 8.1$ & $28.1 \pm 9.9$ & $57.8 \pm 11.3$ \\
\hline & FR\&LFP & $23.0 \pm 3.5$ & $65.7 \pm 5.2$ & $25.0 \pm 4.1$ & $60.5 \pm 6.1$ & $22.9 \pm 4.6$ & $67.3 \pm 8.7$ & $25.0 \pm 4.8$ & $62.7 \pm 10.1$ \\
\hline
\end{tabular}

Table 3. Average (mean \pm standard deviation) of the decoding performance across 10 rats with singleelectrode recording from $\mathrm{S} 1 \mathrm{using}$ the rat-by-rat validation approach.

The results show that the average NRMS decoding errors for pressure and volume decoding were $17.6 \pm 2.7 \%$ and $22.7 \pm 3.4 \%$, respectively, when using the $S 1$ signal, $19.8 \pm 2.4 \%$ and $27.2 \pm 4.6 \%$, respectively, when using the L6 signal, and $14.9 \pm 4.5 \%$ and $19.7 \pm 4.7 \%$, respectively, when the combined signal recorded from S1 and L6 was used for decoding. On average, using two-electrode decoding, an improvement of $21.5 \%$ and $20.4 \%$ was achieved for pressure and volume estimation, respectively, over single-electrode decoding. Almost the same results were observed for the CC. The results of the statistical test show that the two-electrode decoding achieved a lower NRMS error than single-electrode decoding ( $p=0.0318$ for pressure and $p=0.0082$ for volume).

Signal stability. Figure 10 shows the decoding performance for each rat using a trial-by-trial validation approach for single electrode recording. The standard deviation for each rat is very small, which indicates the stability of the decoding across trials.

\section{Discussion}

In this paper, we propose a DRNN for the estimation of bladder pressure and volume from neural activity recorded directly from spinal cord gray matter neurons. The proposed DRNN consists of an autoencoder and a deep LSTM-based decoder. The autoencoder has a deep structure that extracts deep features from input data in an unsupervised manner ${ }^{49}$. The decoding model is based on the LSTM, which has emerged as a general and effective model for capturing long-term temporal dependencies ${ }^{48}$. The LSTM consists of memory cells whose inputs and outputs are controlled by nonlinear gates. Its output not only depends on the current information to perform the estimation but also explicitly takes into account the long-term information from the past.

Since LFP reflects a spatial averaging of synaptic activity in the vicinity of the electrode and carries information that is distinct from spikes, in this paper, we combined modeling of both spiking activity and LFP activity in a unified framework to estimate the pressure and volume of the bladder. The results show that using a combination of FR and LFP can improve decoding performance with respect to using FR or LFP alone.

An important issue in estimating bladder status using neural signals recorded from the spinal cord is the selection of spinal segment that provides more information about the bladder. Afferents innervating the bladder project to the lumbosacral (L6-S1) segments of the rat spinal cord. To address this issue, single-electrode recordings from the L6 and S1 segments were investigated using mutual information and decoding performance. The results show that the S1 segment provides more information about the bladder status than the L6 segment.

Another issue investigated in this study was the effect of two-electrode recoding on decoding performance. For this purpose, a combined signal recorded simultaneously from S1 and L6 was used for decoding, and the results were compared with single-electrode decoding. The results show that the combined signal from both segments provided significantly better decoding performance than the single-electrode decoding. Using an array of electrodes implanted along the L6-S1 segments may improve the decoding performance further. Our results show that average CCs of $78.8 \pm 2.3 \%$ and $83.2 \pm 3.2 \%$ were obtained for pressure estimation using single-electrode and two-electrode decoding, respectively. In contrast, the results of pressure estimation presented in ${ }^{26}$ show that increasing the number of recording channels did not increase the decoding performance. In aforementioned paper, the CC reported for one channel was approximately $75.0 \pm 15.3 \%$, while for 8 channels it was $75.5 \pm 14.6 \%$, using SVR with 30 filter taps. This lack of enhancement may be related to the nonoptimal design of the filter and improper positioning of the electrode tips. Their results show that a CC of approximately $81.8 \pm 10.0 \%$ was obtained for bladder pressure estimation using SVR with 4 channels and 100 filter taps. Regardless of decoding performance, to obtain chronic stability from the spinal recoding, it is necessary to use a multi-electrode array rather than a one- or two-electrode recording system.

An important contribution of the current study is the simultaneous estimation of both the pressure and the volume of the bladder. Simultaneously estimating both the pressure and the volume of the bladder is a critical 

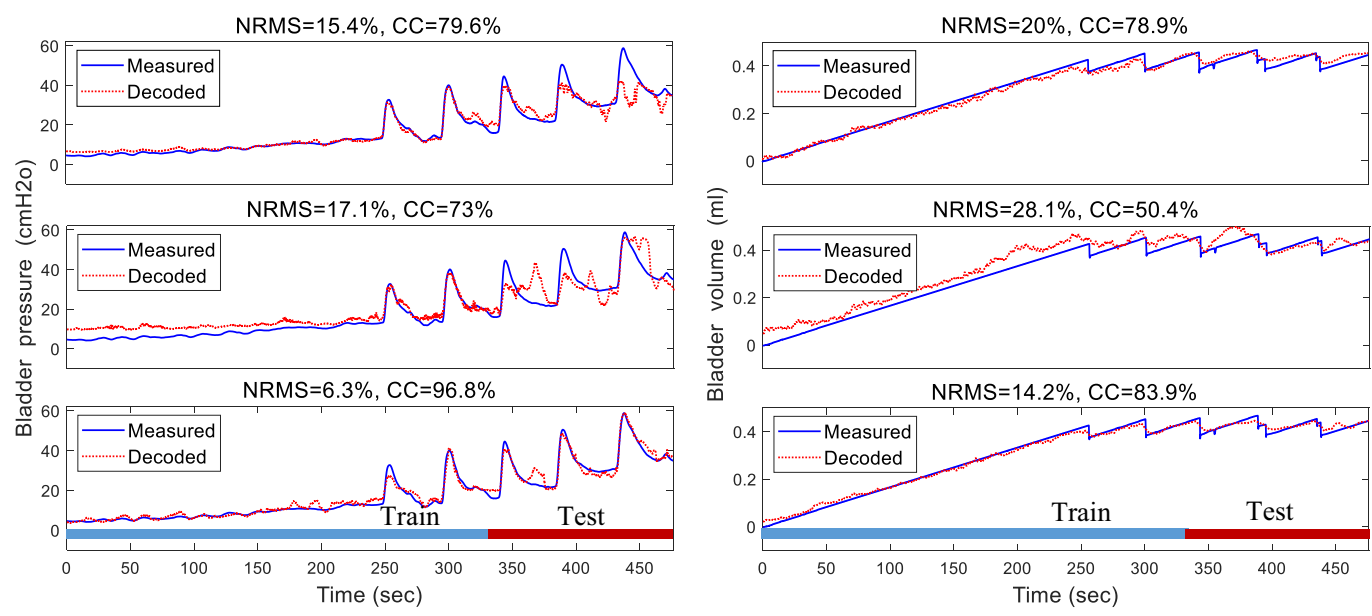

(a)
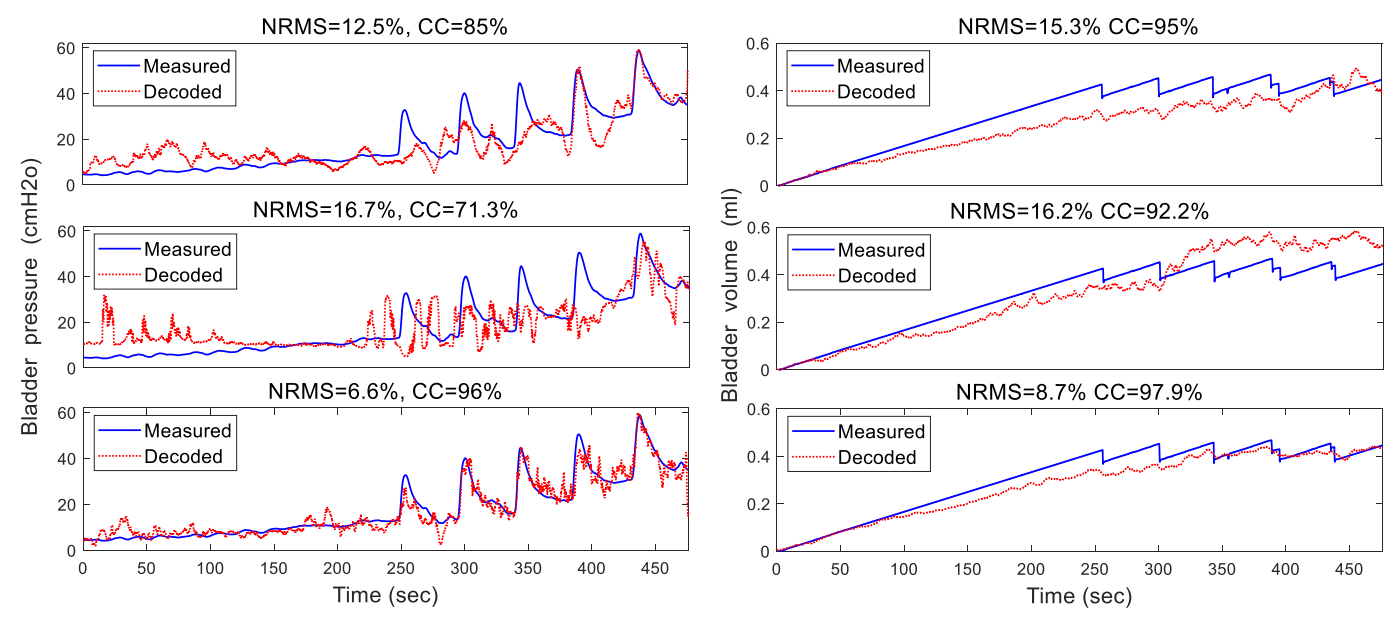

(b)

Figure 9. An example of decoding the bladder pressure (left) and volume (right) during two-electrode recording using inside-trial (a) and trial-by-trial (b) validation approaches (rat 13, trial 2, infusion rate $=6 \mathrm{ml} / \mathrm{h}$ ). Decoding was performed using both FR and the FB5 subband power spectrum extracted from neural signals recorded from S1 (upper trace), L6 (middle trace), and both S1 and L6 (lower trace). During the trial-by-trial validation approach, the network was trained with trial 1 (rat 13 , infusion $\mathrm{rate}=6 \mathrm{ml} / \mathrm{h}$ ) and tested with trial 2 on the same rat (infusion rate $=6 \mathrm{ml} / \mathrm{h}$ ).

\begin{tabular}{|c|c|c|c|c|c|c|c|}
\hline \multirow[b]{2}{*}{ Animal } & \multirow[b]{2}{*}{$\begin{array}{l}\text { Pressure/ } \\
\text { Volume }\end{array}$} & \multicolumn{2}{|l|}{ L6 } & \multicolumn{2}{|l|}{ S1 } & \multicolumn{2}{|l|}{ L6 \& S1 } \\
\hline & & \begin{tabular}{|l|}
$\begin{array}{l}\text { NRMS } \\
(\%)\end{array}$ \\
\end{tabular} & CC (\%) & \begin{tabular}{|l|}
$\begin{array}{l}\text { NRMS } \\
(\%)\end{array}$ \\
\end{tabular} & CC (\%) & \begin{tabular}{|l|}
$\begin{array}{l}\text { NRMS } \\
(\%)\end{array}$ \\
\end{tabular} & CC (\%) \\
\hline \multirow{2}{*}{ Rat 11} & pressure & $18.7 \pm 0.3$ & $75.5 \pm 2.1$ & $18.9 \pm 4.3$ & $78.0 \pm 1.4$ & $12.8 \pm 0.7$ & $80.7 \pm 2.4$ \\
\hline & volume & $30.1 \pm 2.6$ & $61.8 \pm 0.07$ & $24.0 \pm 3.5$ & $69.2 \pm 1.7$ & $20.0 \pm 2.0$ & $70.8 \pm 4.7$ \\
\hline \multirow{2}{*}{ Rat 12} & pressure & $22.0 \pm 2.8$ & $74.7 \pm 1.9$ & $18.0 \pm 1.3$ & $79.7 \pm 0.7$ & $17.9 \pm 0.3$ & $80.1 \pm 1.9$ \\
\hline & volume & $28.0 \pm 0.7$ & $62.2 \pm 0.8$ & $23.1 \pm 0.7$ & $65.5 \pm 2.7$ & $20.5 \pm 0.2$ & $69.1 \pm 1.6$ \\
\hline \multirow{2}{*}{ Rat 13} & pressure & $19.4 \pm 3.8$ & $73.1 \pm 2.6$ & $15.5 \pm 4.2$ & $81.0 \pm 5.6$ & $12.3 \pm 1.4$ & $88.1 \pm 11.1$ \\
\hline & volume & $22.1 \pm 8.4$ & $78.6 \pm 19.2$ & $18.7 \pm 4.8$ & $80.0 \pm 21.2$ & $14.5 \pm 8.2$ & $84.6 \pm 18.5$ \\
\hline \multirow{2}{*}{ Rat 14} & pressure & $18.8 \pm 1.1$ & $69.8 \pm 6.1$ & $18.1 \pm 0.7$ & $76.6 \pm 1.9$ & $15.8 \pm 1.8$ & $82.9 \pm 0.4$ \\
\hline & volume & $27.5 \pm 0.8$ & $58.8 \pm 4.7$ & $24.4 \pm 1.6$ & $68.7 \pm 3.5$ & $21.3 \pm 3.0$ & $75.6 \pm 3.3$ \\
\hline \multirow{2}{*}{ Rat 15} & pressure & $20.1 \pm 1.6$ & $75.6 \pm 0.6$ & $17.7 \pm 1.3$ & $78.8 \pm 0.3$ & $15.7 \pm 1.7$ & $84.6 \pm 0.9$ \\
\hline & volume & $28.5 \pm 0.4$ & $51.0 \pm 0.6$ & $24.0 \pm 0.6$ & $67.1 \pm 0.5$ & $22.6 \pm 0.6$ & $71.0 \pm 0.6$ \\
\hline \multirow{2}{*}{ Average } & pressure & $19.8 \pm 2.5$ & $73.7 \pm 3.8$ & $17.6 \pm 2.6$ & $78.8 \pm 2.3$ & $14.9 \pm 4.8$ & $83.2 \pm 3.2$ \\
\hline & volume & $27.2 \pm 4.7$ & $62.4 \pm 13.5$ & $22.8 \pm 3.5$ & $70.1 \pm 9.9$ & $19.7 \pm 4.7$ & $74.2 \pm 6.2$ \\
\hline
\end{tabular}

Table 4. Average (mean \pm standard deviation) of NRMS and CC from two-electrode recoding using the trialby-trial validation approach. 
(a)

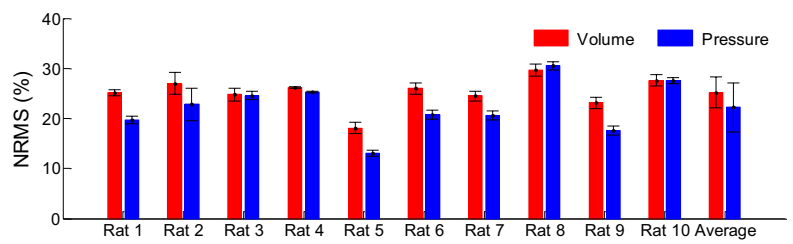

(b)

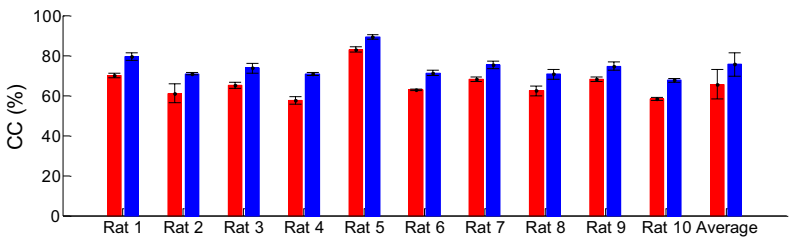

Figure 10. Decoding performance for each rat using the trial-by-trial validation approach. (a) NRMS errors for volume (red) and pressure (blue) estimation. (b) Correlation coefficients for volume (red) and pressure (blue) estimation.

issue in the closed-loop control of the bladder using functional electrical stimulation. The results illustrate that utilizing the proposed deep neural network, the simultaneous estimation of bladder volume and pressure is possible, which had not been investigated in previous studies.

Another issue investigated in this paper is the estimation of pressure/volume for different infusion rates. While the individual is conscious, the bladder filling rate may vary according to different conditions. Different infusion rates lead to different rise times of the bladder pressure (slower or faster). Hence, the decoding model should be generalizable to new conditions. The results of this study show that the proposed deep model is able to estimate the pressure/volume of the bladder for different infusion rates.

The proposed method in this paper has provided very promising results in animal models, but a number of challenges will have to be addressed to establish the clinical use of the method. One of the limitations of the current study is the invasiveness of the measurements, which presents challenges in translating the intraspinal recordings from animal studies to clinical practice. Implanting microelectrodes into the spinal cord can carry the risk of infection and spinal compression. Another challenge facing intraspinal recording is the fabrication and implantation of intraspinal microelectrodes. However, recent progress in microelectrode arrays ${ }^{55-62}$ and knowledge gained from implantable electrodes on animals can be applied to the development of a chronically implanted prosthetic device. Nevertheless, before clinical use of spinal recoding, further tests will be required to assess the long-term mechanical stability of chronically implanted electrodes and the longevity of the chronic recordings. In addition, accurate placement of electrodes in the spinal cord is challenging, even using intraoperative guidance for electrode implantation.

Another critical issue in estimating bladder status using neural signals recorded from the spinal cord is interference from non-bladder signals. It is expected that the decoding model could extract the relevant information about the bladder pressure/volume from neural activity during learning and filter non-bladder signals. However, we did not justify this filtering in this study; this analysis could be considered for a future study. Additionally, further studies should be performed to test the performance of the proposed decoding model in real time for closed-loop control of the bladder.

\section{Data availability}

All datasets generated during the current study are available from the corresponding author upon request.

Received: 16 May 2019; Accepted: 9 November 2019;

Published online: 02 December 2019

\section{References}

1. Fowler, C. J., Griffiths, D. \& Groat, W. C. D. The neural control of micturition. Nat. Rev. Neurosci. 9, 453, https://doi.org/10.1038/ nrn2401 (2008).

2. McGee, M. J., Amundsen, C. L. \& Grill, W. M. Electrical stimulation for the treatment of lower urinary tract dysfunction after spinal cord injury. J. Spinal Cord Med. 38, 135-146, https://doi.org/10.1179/2045772314Y.0000000299 (2015).

3. Chen, G., Liao, L., Dong, Q. \& Ju, Y. The inhibitory effects of pudendal nerve stimulation on bladder overactivity in spinal cord injury dogs: is early stimulation necessary? Neuromodulation: Technology at the Neural Interface. 15, 232-237, https://doi. org/10.1111/j.1525-1403.2012.00434.x (2012).

4. McGee, M. J., Amundsen, C. L. \& Grill, W. M. Temporal pattern of stimulation modulates reflex bladder activation by pudendal nerve stimulation. Neurourol. 35, 882-887, https://doi.org/10.1002/nau.22822 (2016).

5. Wenzel, B. J., Boggs, J. W., Gustafson, K. J. \& Grill, W. M. Closed loop electrical control of urinary continence. J. Urol. 175, 1559-1563, https://doi.org/10.1016/S0022-5347(05)00657-9 (2006).

6. Opisso, E., Borau, A. \& Rijkhoff, N. J. M. Urethral sphincter EMG-controlled dorsal penile/clitoral nerve stimulation to treat neurogenic detrusor overactivity. J. Neural Eng. 8, 036001, https://doi.org/10.1088/1741-2560/8/3/036001 (2011).

7. Bhadra, N., Bhadra, N., Kilgore, K. \& Gustafson, K. J. High frequency electrical conduction block of the pudendal nerve. J. Neural Eng. 3, 180, https://doi.org/10.1088/1741-2560/3/2/012 (2006).

8. Tai, C., Wang, J., Wang, X., Roppolo, J. R. \& Groat, W. C. Voiding reflex in chronic spinal cord injured cats induced by stimulating and blocking pudendal nerves. Neurourol. Urodyn: Official Journal of the International Continence Society. 26, 879-886, https://doi. org/10.1002/nau.20430 (2007). 
9. Boger, A., Bhadra, N. \& Gustafson, K. J. Bladder voiding by combined high frequency electrical pudendal nerve block and sacral root stimulation. Neurourol Urodyn: Official Journal of the International Continence Society. 27, 435-439, https://doi.org/10.1002/ nau.20538 (2008)

10. Boger, A. S., Bhadra, N. \& Gustafson, K. J. High frequency sacral root nerve block allows bladder voiding. Neurourol. 31, 677-682, https://doi.org/10.1002/nau.21075 (2012).

11. Peh, W. Y. X. et al. Novel neurostimulation of autonomic pelvic nerves overcomes bladder-sphincter dyssynergia. Front. Neurosci. 12, 186, https://doi.org/10.3389/fnins.2018.00186 (2018)

12. Kirkham, A. P. S., Shah, N. C., Knight, S. L., Shah, P. J. R. \& Craggs, M. D. The acute effects of continuous and conditional neuromodulation on the bladder in spinal cord injury. Spinal cord. 39, 420, https://doi.org/10.1038/sj.sc.3101177 (2001).

13. Dalmose, A. L. et al. Conditional stimulatzion of the dorsal penile/clitoral nerve may increase cystometric capacity in patients with spinal cord injury. Neurourol. 22, 130-137, https://doi.org/10.1002/nau.10031 (2003).

14. Hansen, J. et al. Treatment of neurogenic detrusor overactivity in spinal cord injured patients by conditional electrical stimulation. J. Urol. 173, 2035-2039, https://doi.org/10.1097/01.ju.0000158160.11083.1b (2005).

15. Horvath, E. E., Yoo, P. B., Amundsen, C. L., Webster, G. D. \& Grill, W. M. Conditional and continuous electrical stimulation increase cystometric capacity in persons with spinal cord injury. Neurourol Urodyn: Official Journal of the International Continence Society. 29, 401-407, https://doi.org/10.1002/nau.20766 (2010).

16. Brouillard, C. B., Crook, J. J., Irazoqui, P. P. \& Lovick, T. A. Suppression of urinary voiding by conditional high frequency stimulation of the pelvic nerve in conscious rats. Front. Physiol. 9, 437, https://doi.org/10.3389/fphys.2018.00437 (2018).

17. Melgaard, J. \& Rijkhoff, N. J. M. Detecting the onset of urinary bladder contractions using an implantable pressure sensor. IEEE Trans. Neural syst. Rehabil. Eng. 19, 700-708, https://doi.org/10.1109/TNSRE.2011.2171368 (2011).

18. Majerus, S. J., Fletter, P. C., Damaser, M. S. \& Garverick, S. L. Low-power wireless micromanometer system for acute and chronic bladder-pressure monitoring. IEEE Trans. Biomed. Eng. 58, 763-767, https://doi.org/10.1109/TBME.2010.2085002 (2011).

19. Lee, D. S. et al. Real-time bladder volume monitoring by the application of a new implantable bladder volume sensor for a small animal model. Kaohsiung J. Med. Sci. 27, 132-137, https://doi.org/10.1016/j.kjms.2010.12.006 (2011).

20. Lee, H. Y. et al. Sensitivity-enhanced LC pressure sensor for wireless bladder pressure monitoring. IEEE Sens. J. 16, 4715-4724, https://doi.org/10.1109/JSEN.2016.2533262 (2016)

21. Majerus, S. J. et al. Suburothelial bladder contraction detection with implanted pressure sensor. PloS one. 12, e0168375, https://doi. org/10.1371/journal.pone.0168375 (2017)

22. Weber, M. J. et al. A Miniaturized single-transducer implantable pressure sensor with time-multiplexed ultrasonic data and power links. CIRC. 53, 1089-1101, https://doi.org/10.1109/JSSC.2017.2782086 (2018).

23. Stauffer, F. et al. Soft electronic strain sensor with chipless wireless readout: toward real-time monitoring of bladder volume. Adv. Mater. Technol. 3, 1800031, https://doi.org/10.1002/admt.201800031 (2018).

24. Abelson, B. et al. Ambulatory urodynamic monitoring: state of the art and future directions. Nat. Rev. Urol. https://doi.org/10.1038/ s41585-019-0175-5 (2019).

25. Yu, L., Kim, B. \& Meng, E. Chronically implanted pressure sensors: challenges and state of the field. Sensors. 14, 20620-44, https:// doi.org/10.3390/s141120620 (2014).

26. Im, C. et al. Decoding intravesical pressure from local field potentials in rat lumbosacral spinal cord. J. Neural Eng. 13, 056005, https://doi.org/10.1088/1741-2560/13/5/056005 (2016).

27. Ross, S. E., Ouyang, Z., Rajagopalan, S. \& Bruns, T. M. Evaluation of decoding algorithms for estimating bladder pressure from dorsal root ganglia neural recordings. ANN. Biomed. Eng. 46, 233-246, https://doi.org/10.1007/s1043 (2018).

28. Hansen, J. et al. Urethral sphincter EMG as event detector for neurogenic detrusor overactivity. IEEE Trans. Biomed. Eng. 54, 1212-1219, https://doi.org/10.1109/TBME.2007.890739 (2007).

29. Knight, S. L., Edirisinghe, N., Leaker, B., Susser, J. \& Craggs, M. D. Conditional neuromodulation of neurogenic detrusor overactivity using transrectal stimulation in patients with spinal cord injury: A proof of principle study. Neurourol. 37, 385-393, https://doi. org/10.1002/nau.23310 (2018).

30. Rutter, E. M. et al. Detection of bladder contractions from the activity of the external urethral sphincter in rats using sparse regression. IEEE Trans. Neural Syst. Rehabil. Eng. 26, 1636-1644, https://doi.org/10.1109/TNSRE.2018.2854675 (2018).

31. Wenzel, B. J., Boggs, J. W., Gustafson, K. J. \& Grill, W. M. Detecting the onset of hyper-reflexive bladder contractions from the electrical activity of the pudendal nerve. IEEE Trans. Neural syst. Rehabil. Eng. 13, 428-435, https://doi.org/10.1109/ TNSRE.2005.848355 (2005)

32. Mathews, K. S. et al. Acute monitoring of genitourinary function using intrafascicular electrodes: selective pudendal nerve activity corresponding to bladder filling, bladder fullness, and genital stimulation. Urology. 84, 722-729, https://doi.org/10.1016/j. urology.2014.05.021 (2014).

33. Jezernic, S., Wen, J. G., Rijkhoff, N. J., Djurhuus, J. C. \& Sinkjaer, T. Analysis of bladder related nerve cuff electrode recordings from preganglionic pelvic nerve and sacral roots in pigs. J. Urol. 163, 1309-1314, https://doi.org/10.1016/S0022-5347(05)67769-5 (2000).

34. Kurstjens, G. A. M., Borau, A., Rodriguez, A., Rijkhoff, N. J. M. \& Sinkjaer, T. Intraoperative recording of electroneurographic signals from cuff electrodes on extradural sacral roots in spinal cord injured patients. J. Urol. 174, 1482-1487, https://doi. org/10.1097/01.ju.0000173005.70269.9c (2005).

35. Bruns, T. M., Gaunt, R. A. \& Weber, D. J. Multielectrode array recordings of bladder and perineal primary afferent activity from the sacral dorsal root ganglia. J. Neural Eng. 8, 056010, https://doi.org/10.1088/1741-2560/8/5/056010 (2011).

36. Mendez, A., Sawan, M., Minagawa, T. \& Wyndaele, J. J. Estimation of bladder volume from afferent neural activity. IEEE Trans. Neural syst. Rehabil. Eng. 21, 704-715, https://doi.org/10.1109/TNSRE.2013.2266899 (2013).

37. Weber, D. J., Stein, R. B., Everaert, D. G. \& Prochazka, A. Limb-state feedback from ensembles of simultaneously recorded dorsal root ganglion neurons. J. Neural Eng. 4, S168, https://doi.org/10.1088/1741-2560/4/3/S04 (2007).

38. Stein, R. B. et al. Coding of position by simultaneously recorded sensory neurones in the cat dorsal root ganglion. J. Physipl. 560, 883-896, https://doi.org/10.1113/jphysiol.2004.068668 (2004).

39. Wagenaar, J. B., Ventura, V. \& Weber, D. J. State-space decoding of primary afferent neuron firing rates. J. Neural Eng. 8, 016002, https://doi.org/10.1088/1741-2560/8/1/016002 (2011).

40. Rigosa, J., Weber, D. J., Prochazka, A., Stein, R. B. \& Micera, S. Neuro-fuzzy decoding of sensory information from ensembles of simultaneously recorded dorsal root ganglion neurons for functional electrical stimulation applications. J. Neural Eng. 8, 046019, https://doi.org/10.1088/1741-2560/8/4/046019 (2011).

41. Weber, D. J., Stein, R. B., Everaert, D. G. \& Prochazka, A. Decoding sensory feedback from firing rates of afferent ensembles recorded in cat dorsal root ganglia in normal locomotion. IEEE Trans. Neural syst. Rehabil. Eng. 14, 240-243, https://doi.org/10.1109/ TNSRE.2006.875575 (2006)

42. Umeda, T. et al. Decoding of the spike timing of primary afferents during voluntary arm movements in monkeys. Front. Neurosci. 8, 97, https://doi.org/10.3389/fnins.2014.00097 (2014)

43. Bruns, T. M., Wagenaar, J. B., Bauman, M. J., Gaunt, R. A. \& Weber, D. J. Real-time control of hind limb functional electrical stimulation using feedback from dorsal root ganglia recordings. J. Neural Eng. 10, 026020, https://doi.org/10.1088/17412560/10/2/026020 (2013)

44. Holinski, B. J., Everaert, D. G., Mushahwar, V. K. \& Stein, R. B. Real-time control of walking using recordings from dorsal root ganglia. J. Neural Eng. 10, 056008, https://doi.org/10.1088/1741-2560/10/5/056008 (2013). 
45. Han, S., Chu, J. U., Kim, H., Park, J. W. \& Youn, I. Multiunit activity-based real-time limb-state estimation from dorsal root ganglion recordings. Sci. Rep-UK. 7, 44197, https://doi.org/10.1038/srep44197 (2017).

46. Khurram, A. et al. Chronic monitoring of lower urinary tract activity via a sacral dorsal root ganglia interface. J. Neural Eng. 14, 036027, https://doi.org/10.1088/1741-2552/aa6801 (2017).

47. Park, J. H. et al. Detecting bladder fullness through the ensemble activity patterns of the spinal cord unit population in a somatovisceral convergence environment. J. Neural Eng. 10, 056009, https://doi.org/10.1088/1741-2560/10/5/056009 (2013).

48. Hochreiter, S. \& Schmidhuber, J. Long short-term memory. Neural Comput. 9, 1735-1780, https://doi.org/10.1162/ neco.1997.9.8.1735 (1997).

49. Chen, Y., Lin, Z., Zhao, X., Wang, G. \& Gu, Y. Deep learning-based classification of hyperspectral data. IEEE J. Sel. Topics Appl. Earth Observ. Remote Sens. 7, 2094-2107, https://doi.org/10.1109/JSTARS.2014.2329330 (2014).

50. Greff, K., Srivastava, R. K., Koutník, J., Steunebrink, B. R. \& Schmidhuber, J. LSTM: A search space odyssey. IEEE Trans. Neural Netw. Learn. Syst. 28, 2222-2232, https://doi.org/10.1109/TNNLS.2016.2582924 (2017).

51. Patterson, J. \& Gibson, A. Deep Learning: A Practitioner's Approach, First edition (2017).

52. Tsoi, A. C. \& Back, A. D. Locally recurrent globally feedforward networks: a critical review of architectures. IEEE Trans. Neural Netw. 5, 229-239, https://doi.org/10.1109/72.279187 (1994).

53. Hochreiter, S., Bengio, Y., Frasconi, P. \& Schmidhuber, J. Gradient Flow in Recurrent Nets: the Difficulty of Learning Long-Term Dependencies (2001).

54. Darbellay, G. A. \& Vajda, I. Estimation of the information by an adaptive partitioning of the observation space. IEEE Trans. Inf. Theory. 45, 1315-1321, https://doi.org/10.1109/18.761290 (1999).

55. Toossi, A., Everaert, D. G., Azar, A., Dennison, C. R. \& Mushahwar, V. K. Mechanically stable intraspinal microstimulation implants for human translation. ANN. Biomed. Eng. 1;45(3):681-94, https://doi.org/10.1007/s10439-016-1709-0 (2017).

56. Debnath, S., Bauman, M. J., Fisher, L. E., Weber, D. J. \& Gaunt, R. A. Microelectrode array recordings from the ventral roots in chronically implanted cats. Front. Neurol. Neurosci. 5, 104, https://doi.org/10.3389/fneur.2014.00104 (2014).

57. Ersen, A., Elkabes, S., Freedman, D. S. \& Sahin, M. Chronic tissue response to untethered microelectrode implants in the rat brain and spinal cord. J. Neural Eng. 12, 016019, https://doi.org/10.1088/1741-2560/12/1/016019 (2015).

58. Kim, T., Branner, A., Gulati, T. \& Giszter, S. F. Braided multi-electrode probes: mechanical compliance characteristics and recordings from spinal cords. J. Neural Eng. 10, 045001, https://doi.org/10.1088/1741-2560/10/4/045001 (2013).

59. Kipke, D. R., Vetter, R. J., Williams, J. C. \& Hetke, J. F. Silicon-substrate intracortical microelectrode arrays for long-term recording of neuronal spike activity in cerebral cortex. IEEE Trans. Neural syst. Rehabil. Eng. 11, 151-5, https://doi.org/10.1109/ TNSRE.2003.814443 (2003).

60. Moxon, K. A., Leiser, S. C., Gerhardt, G. A., Barbee, K. A. \& Chapin, J. K. Ceramic-based multisite electrode arrays for chronic single-neuron recording. IEEE Trans. Biomed. Eng. 51, 647-566, https://doi.org/10.1109/TBME.2003.821037, (2004).

61. Rousche, P. J. \& Normann, R. A. Chronic recording capability of the Utah Intracortical Electrode Array in cat sensory cortex. J. Neurosci. Methods. 82, 1-15, https://doi.org/10.1016/S0165-0270(98)00031-4 (1998).

62. Zhong, Y. \& Bellamkonda, R. V. Dexamethasone-coated neural probes elicit attenuated inflammatory response and neuronal loss compared to uncoated neural probes. Brain Res. 1148, 15-27, https://doi.org/10.1016/j.brainres.2007.02.024 (2007).

\section{Acknowledgements}

This work was supported by the Iran Neural Technology Research Centre, Tehran, Iran under Grant 48.M.111194. The authors would like to thank the members of Iran Neural Technology Research Centre for their assistance during the experiments and preparing the facilities for conducting the experiments and data analysis.

\section{Author contributions}

A.E. and M.J. contributed to the design of experiments. M.J. conducted the experiments. M.J. analyzed the data. Manuscript preparation was contributed jointly by all authors.

\section{Competing interests}

The authors declare no competing interests.

\section{Additional information}

Correspondence and requests for materials should be addressed to A.E.

Reprints and permissions information is available at www.nature.com/reprints.

Publisher's note Springer Nature remains neutral with regard to jurisdictional claims in published maps and institutional affiliations.

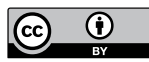

Open Access This article is licensed under a Creative Commons Attribution 4.0 International License, which permits use, sharing, adaptation, distribution and reproduction in any medium or format, as long as you give appropriate credit to the original author(s) and the source, provide a link to the Creative Commons license, and indicate if changes were made. The images or other third party material in this article are included in the article's Creative Commons license, unless indicated otherwise in a credit line to the material. If material is not included in the article's Creative Commons license and your intended use is not permitted by statutory regulation or exceeds the permitted use, you will need to obtain permission directly from the copyright holder. To view a copy of this license, visit http://creativecommons.org/licenses/by/4.0/.

(C) The Author(s) 2019 